\title{
Couragierte Pionierinnen - Botanikerinnen im 18. Jahrhundert
}

\author{
Thomas Pfister \\ Gartentherapie Pfister GmbH, Zürich, Schweiz \\ Schlüsselwörter \\ Botanik · Geschichte · Jeanne Baret · Gender
}

\section{Zusammenfassung}

Botanik als Wissenschaft war bis in das 18. Jahrhundert ein Teilgebiet der Medizin und den Männern vorbehalten. Trotzdem wagten sich einige mutige Pionierinnen in diese Domäne. Als Pflanzensammlerinnen oder -zeichnerinnen und als «Forscherinnen» leisteten sie wichtige Erkenntnisse zur Entwicklung der Botanik. Als Beispiel einer Pionierin der Botanik wird die Geschichte der Jeanne Baret erzählt die als erste Frau die Welt umrundete und dabei unglaubliche Leistungen zur Entdeckung noch unbekannter Pflanzen erbrachte. Während alle Welt den Botaniker Carl Linné kennt, sind die frühen Botanikerinnen der Nachwelt oft kaum (mehr) bekannt. Der Essay vergleicht die speziellen Merkmale dieser Frauen, die es ihnen ermöglichten, trotz widriger Umstände einen Beitrag zur Botanik zu leisten. Neben einer starken Persönlichkeit waren dies bei allen Botanikerinnen viel Enthusiasmus für ihr Fachgebiet sowie die Unterstützung durch männliche Förderer in ihrer nächsten Umgebung.

\section{Keywords}

Botany $\cdot$ History $\cdot$ Jeanne Baret $\cdot$ Gender

\section{Summary}

Courageous Pioneers - Female Botanists in the 18th Century

Until the 18th century, scientific botany was a part of medicinal science and therefore reserved for men only. A few courageous female pioneers still entered this field: as plant collectors, plant illus-

Überarbeitete und aktualisierte Version einer Projektarbeit im Rahmen des CAS «Ethnobotanik und Ethnomedizin» an der Universität Zürich, Schweiz. trators, and researchers these women contributed to the development of botany. The biography of Jeanne Baret, who circumnavigated the globe as the very first woman, is an impressive example of an early botanist. Whereas the scientific merits of Carl Linné are well known, early female botanists sank into oblivion. This essay compares specific characteristics of early female botanists in unfavourable conditions. Beside a strong personality, all these early botanists showed great enthusiasm for their field and had a male promoter in their immediate vicinity.

\section{Mots clés}

Botanique $\cdot$ Histoire $\cdot$ Jeanne Baret $\cdot$ Sexe

\section{Résumé}

\section{Courageuses pionnières: les femmes botanistes du XVIIle siècle}

Même si la botanique en tant que science était un domaine de la médecine réservé aux hommes jusqu'au début du XVIIIe siècle, quelques courageuses pionnières s'y sont aventurées. En tant que cueilleuses ou dessinatrices des plantes et en tant que «chercheuses», elles ont apporté des connaissances importantes au développement de la botanique. L'histoire de Jeanne Baret nous est racontée comme étant un exemple de pionnière dans ce domaine: elle fut la première femme à avoir fait le tour du monde et elle a apporté une incroyable contribution au développement de plantes encore inconnues. Alors que toute la planète connaît le botaniste Carl Linné, les premières femmes botanistes sont peu (ou plus) connues de la postérité. Cet essai compare le caractère spécial de ces femmes qui, malgré des conditions défavorables, leur a permis de faire avancer la botanique. En plus d'une forte personnalité, toutes ces botanistes faisaient preuve d'un grand enthousiasme pour leur domaine de spécialité et bénéficiaient du soutien des chercheurs masculins dans leur environnement proche.

\section{KARGER \\ Fax +497614520714 Information@Karger.com} www.karger.com
Thomas Pfister

Psychologe lic. phil., Gartentherapeut

Gartentherapie Pfister GmbH

Ackersteinstrasse 67, 8049 Zürich, Schweiz

thomas.pfister-adm@bluewin.ch

www.gartenundtherapie.ch 


\section{Mutige Pionierinnen}

Während der Zeit des CAS «Ethnobotanik und Ethnomedizin» an der Universität Zürich wurde im Tagesanzeiger ein Artikel publiziert, der die Geschichte von Jeanne Baret erzählte, die im 18. Jahrhundert als Mann verkleidet rund um die Welt reiste [1]. Recherchen brachten einige weitere Botanikerinnen ans Tageslicht, die ebenfalls Pionierarbeit in diesem von Männern dominierten Feld vollbracht hatten [2]. Der folgende Essay stellt die ausserordentlichen Leistungen dieser frühen Botanikerinnen ins Zentrum und versucht, ihre speziellen Persönlichkeitsmerkmale und Eigenschaften zu beleuchten.

\section{Eine kurze Geschichte der Botanik}

Botanik ist die Wissenschaft von den Pflanzen. «Von ihr werden Bau, Lebensweise, Fortpflanzung, Veränderlichkeit, Anpassung und natürliche Verbreitung der Pflanzen erforscht. Die Botanik gliedert sich in: Morphologie, Anatomie, Physiologie, Ökologie, Systematik (= Taxonomie), Pflanzengeographie (Vegetationskunde und Chronologie), Paläobotanik» [3, S. 134].

\section{Antike und Mittelalter}

Aristoteles (384-322 v. Chr.) wird als Begründer der wissenschaftlichen Pflanzenkunde betrachtet. Sein Schüler Theophrastus von Eresos (370-285 v. Chr.) schrieb ein erstes «wissenschaftliches» Werk über Pflanzen. Im 1. Jahrhundert nach der Zeitwende erarbeitete der griechische Arzt Pedanios Dioskurides mit rund 500 detailliert beschriebenen Pflanzenarten die umfangreichste Pflanzenliste des Altertums. Er prägte den Begriff «Botanik», der vom griechischen Begriff «botane» stammt, was übersetzt Futterkraut, Gewächs oder Gras bedeutet. Karl der Grosse (747-814) katalogisierte in seinem Gesetz «Capitulare de villis» um das Jahr 812 Gemüsepflanzen, Gewürze sowie Arznei- und Zierpflanzen, die fortan in jedem Gutsbetrieb angebaut werden mussten. Hildegard von Bingen (1098-1179) fügte zu einer Liste von 300 Gewächsen im lateinischen Text die deutschen Bezeichnungen hinzu. Albertus Magnus (ca. 1200-1280) schrieb unter dem Titel «De vegetabilibus libri» eine siebenbändige Einführung in die Botanik. 1485 erschien von Johann Wonnecke von Kaub unter dem Titel «Gart der Gesundheit» eines der ersten Kräuterbücher [4].

\section{0 bis 1700}

Conrad Gessner (1516-1565), ein Schweizer Naturforscher, beschäftigte sich als einer der Ersten mit Blüten und Fruchtteilen und untersuchte die Vegetation in den unterschiedlichen Höhenzonen. Im 16. Jahrhundert erschienen verschiedene Kräuterbücher, so z.B. von Otto Brunfels (1532-1537), Hieronymus Bock (1539), Leonhart Fuchs (1542), Adam Lonitzer (1557) oder Rembert Dodoens (1583).

Im 17. Jahrhundert erschienen verschiedene FloraWerke, z.B. die Flora von Altdorf von Ludwig Jungermann (1615), die Flora von Danzig von Nicolaus Oelhafen (1643) oder die Flora der Pfalz von Georg Franck von Franckenau (1680).

Caspar Bauhin veröffentlichte 1623 ein monumentales Werk mit allen damals bekannten ca. 6000 Pflanzen. Im 17. Jahrhundert erschienen die ersten Publikationen zur Pflanzenernährung und zur Einteilung der Pflanzen anhand von Blütenmerkmalen. Erste Untersuchungen unter dem Mikroskop zeigten Details der Anatomie von Pflanzen. John Ray publizierte mit der «Synopsis methodica» neue Ansätze zur Pflanzensystematik. August Quirinius Rivinus entwickelte einen ersten Ansatz zu einer binären Nomenklatur [4].

\section{Botanik im 18. Jahrhundert}

Die Botanik war bis in das 18. Jahrhundert ein Teilgebiet der Medizin und wurde an denselben Lehrstühlen unterrichtet. Auch die Pharmazie blieb lange sehr eng mit der Medizin verbunden. Eigene Institute für Botanik entstanden erst im 19. Jahrhundert [5]. Somit waren fast alle im 18. Jahrhundert tätigen Botaniker, wie z.B. Carl von Linné oder Philibert Commerson, auch Ärzte. Frauen waren bis weit in das 19. Jahrhundert hinein von allen Studien ausgeschlossen, so auch von der Medizin. Ausnahmen wie beispielsweise Dorothea Erxleben, die 1754 als Ärztin promovierte, bestätigen diese Regel. In Österreich war Gabriele Possaner 1897 die erste Frau, die als Medizinerin promovierte [6].

Als wichtige Erkenntnisse der Botanik im 18. Jahrhundert wurden erste Kreuzungen von Pflanzen vollzogen und Erkenntnisse zur Fortpflanzung der Pflanzen veröffentlicht. Im Weiteren wurden die Fotosynthese sowie verschiedene pflanzenphysiologische Prozesse wie z.B. das Saftsteigen in Bäumen entdeckt. Schliesslich machte man Fortschritte in der Etablierung einer Systematik des Pflanzenreichs. Carl von Linné publizierte 1735 sein wegweisendes Werk «Systema naturae» [4].

\section{Wo sind die Botanikerinnen?}

Auf Wikipedia gibt es eine Liste mit 347 Botanikern aus dem 18. Jahrhundert. Darunter ist nur eine (!) Frau zu finden: Catharina Helena Dörrien [7]. Durch weitere Recherchen konnten insgesamt sieben Botanikerinnen aus dem 18. Jahrhundert ausfindig gemacht werden. 


\section{Sind das überhaupt Botanikerinnen?}

Diese Frage hängt sehr stark mit den Kriterien zusammen, die man dabei anwendet. Ein erstes Kriterium stellt ein abgeschlossenes Botanikstudium dar. Wie oben dargestellt, war die Botanikim 18. Jahrhundert noch kein eigenes Fach, sondern ein Teil der Medizin. Da damals Frauen zum Medizinstudium nicht zugelassen waren, konnten sie keine Vorlesungen in Botanik besuchen. Die sieben «Botanikerinnen» eigneten sich ihr Wissen auf ganz unterschiedliche Weise an: Jeanne Baret erwarb sich ihre Pflanzenkenntnisse von ihrer Mutter und dann in der Zusammenarbeit mit ihrem Partner, dem Botaniker Philibert Commerson. Anna Blackburne und Catharina Helena Dörrien wurden von ihren Vätern in Naturwissenschaft unterrichtet. Elizabeth Blackwell und Maria Sibylla Merian erhielten eine künstlerische, aber keine naturkundlich-botanische Ausbildung. Karoline Luise von Baden und Sophie Charlotte von MecklenburgStrelitz erhielten im Rahmen ihrer höfischen Erziehung neben vielen anderen Fächern auch Unterricht in Botanik.

Ein zweites Kriterium ist das Selbstverständnis als Botanikerin. Jeanne Baret muss wohl eher als «Kräuterfrau» denn als Botanikerin bezeichnet werden. Während ihrer langjährigen Zusammenarbeit mit dem Botaniker Philibert Commerson erwarb sie sich aber ein grosses zusätzliches Wissen über Pflanzen. Anna Blackburne stand in Briefwechsel mit verschiedenen Wissenschaftlern, so auch mit Carl von Linné. Sie gliederte ihre grosse naturkundliche Sammlung nach den neuesten wissenschaftlichen Erkenntnissen. Elisabeth Blackwell verstand sich als Pflanzenzeichnerin. Ihre rund 500 detailgetreuen Abbildungen von Medizinalpflanzen wurden als zweibändiges Buch herausgegeben. Maria Sibylla Merian verstand sich zuerst als Künstlerin. Mit der von ihr entwickelten Kombination von naturgetreuen Zeichnungen und beschreibenden Texten beschritt sie Neuland und beeindruckte die Fachwelt. Ihre Expeditionen in Südamerika machten sie zur angesehenen Forscherin auf dem Gebiet der Insekten- und Pflanzenkunde. Karoline Luise von Baden verstand sich zuerst als Landesherrin. Daneben war sie eine tüchtige Geschäftsfrau und Unternehmerin. Ihr grosses Naturalienkabinett gliederte sie nach den neuesten Ordnungssystemen. Catharina Helena Dörrien sah sich auf dem Gebiet der Botanik als Wissenschaftlerin und erhielt auch dementsprechende Anerkennung [8].

Als drittes Kriterium dient die Anerkennung in Fachkreisen. Das wohl schönste Kompliment erhielt Karoline Luise von Baden: «In der Botanik ist sie so starck wie ein Professor» [9, S. 328]. Sophie Charlotte von MecklenburgStrelitz erhielt den Ehrentitel «Königin der Botanik», weil sie sich um den Garten Kew in der Nähe von London verdient gemacht hatte. Maria Sibylla Merian war als
Pflanzen- und Insektenmalerin in ihrer Zeit sehr angesehen. Da sie sich dabei viele künstlerische Freiheiten nahm und auch bei den Texten nicht immer ganz genau war, war sie aber in wissenschaftlichen Kreisen umstritten. Catharina Helena Dörrien wurde zuerst durch ihre pädagogischen Schriften bekannt. Ihre Ehrenmitgliedschaften in verschiedenen botanischen Gesellschaften verdankte sie ihrem 1777 veröffentlichten wissenschaftlichen Katalog aller in ihrer Heimat vorkommenden Pflanzen. Auch ihre rund 1400 Pflanzenzeichnungen trugen wesentlich zu ihrem guten Ruf in der Fachwelt bei. Karoline Luise von Baden wurde mit ihrem grossen Naturalienkabinett berühmt. Bekannte Naturforscher standen mit ihr in regem Austausch und ermöglichten ihr, das Kabinett zu erweitern. Die über 500 Abbildungen von Medizinalpflanzen von Elisabeth Blackwell erregten in Fachkreisen grosses Aufsehen, so unter anderem in Apothekerkreisen und beim Direktor des botanischen Gartens von Chelsea. Die naturgetreuen Pflanzenabbildungen wurden als zweibändiges Buch publiziert - damals keine Selbstverständlichkeit. Anna Blackburne stand in regelmässigem Kontakt mit verschiedenen Wissenschaftlern wie z.B. Carl von Linné. Der damals bekannte Naturforscher Thomas Pennant lobte sie für ihr umfangreiches Naturmuseum und ihre Beiträge über die Erforschung der nordamerikanischen Vögel. Jeanne Baret ging in die Geschichte ein als erste Frau, welche die Welt umsegelte. Da sie keinerlei schriftliche Zeugnisse hinterliess, kann ihr Beitrag zur Botanik im 18. Jahrhundert nur schwer abgeschätzt werden. Sie sammelte auf ihrer langen Reise zusammen mit Philibert Commerson Tausende von Pflanzen, die zum Teil Eingang in das Nationale Französische Herbar fanden [2, S. 32-33].

\section{Eine kurze Biografie der Jeanne Baret}

\section{Die Kräuterfrau und der Botaniker}

Jeanne Baret (Abb. 1) kam am 27. Juli 1740 als Tochter eines Tagelöhners und einer Kräuterfrau im französischen Lome zur Welt. Die Familie lebte in sehr ärmlichen Verhältnissen. In ihrem Elternhaus hat Jeanne Baret trotzdem das Lesen gelernt. Sehr wahrscheinlich wurde sie von ihrer Mutter im Sammeln, Aufbewahren und Anwenden von Heilpflanzen unterrichtet, die damals für die ärmere Bevölkerung die einzige Medizin darstellten. Kräuterfrauen lieferten ihr Sammelgut an Apotheker und Ärzte. Sie besassen grosse Kenntnisse über Pflanzen und hatten täglich mit ihnen zu tun. Das Wissen wurde von der Mutter an die Töchter weitergegeben [10, S. 13-28].

Philibert Commerson, 1728 geboren, entstammte einer bürgerlichen Handelsfamilie aus Lyon. Er gehörte der Mittelklasse an, und die Familie besass Ländereien. Er besuchte die örtliche Klosterschule und trat dann in 
das Jesuitenkollegium von Bourg-en-Bresse ein. Vom Vater beeinflusst, dessen Hobby die Naturphilosophie war, entstand eine lebenslange Liebe zur Botanik. Schon mit 15 Jahren hatte er ein umfangreiches Herbarium angelegt. Er besuchte auch regelmässig den Botanischen Garten der Universität von Montpellier. Auf seinen Exkursionen war er in den Pyrenäen und im französisch-schweizerischen Jura unterwegs. 1760 heiratete er Antoinette-Vivante Beau. Im selben Jahr traf er sehr wahrscheinlich bei seinen Streifzügen zum ersten Mal Jeanne Baret, die ihm eine Liste mit Medizinalpflanzen schenkte - das einzige schriftliche Zeugnis von Jeanne Baret! Sie fühlte sich geschmeichelt, dass ein so gebildeter Mann sich für sie und ihre Pflanzen interessierte. Er stellte sie 1764 als Haushälterin an und hatte so eine Lehrerin, Assistentin und Liebhaberin [9, S. 29-41].

\section{Ein Identitätswechsel in Paris}

Die Reise nach Paris war für die schwangere Jeanne Baret ziemlich anstrengend. In Paris sah sie innerhalb weniger Tage mehr als in den bisherigen Jahren ihres Lebens. Sie ging in den Botanischen Garten und in die königlichen Gärten. Im Dezember 1764 schenkte sie ihrem Sohn Jean-Pierre das Leben. Da Commerson mit dem Kind aber aus Standesgründen nichts zu tun haben wollte, gab sie es in ein Waisenhaus, wo es ein Jahr später starb. Sie übernahm nun ganz die Haushaltführung in Paris [10, S. 42-56].

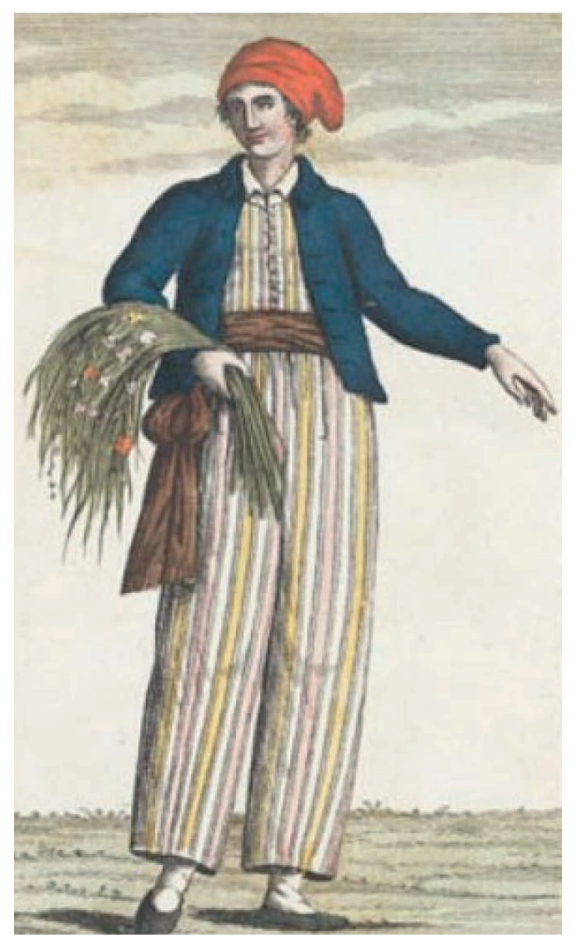

Abb. 1.

Einziges Porträt von Jeanne Baret.
Commerson wurde bezüglich der Teilnahme an einer Weltumsegelung angefragt, um nutzbare tropische Pflanzen zu finden. Da auf französischen Kriegsschiffen keine Frauen erlaubt waren, entstand der Plan, sie als Mann verkleidet als seinen Gehilfen bei der Expedition zu engagieren. Sie trainierte Muskelkraft, lernte tiefer $\mathrm{zu}$ sprechen und machte erste Versuche mit Bandagen um ihre Brüste [10, S. 57-71].

\section{Das Überqueren der Linie}

Auf ihrem Schiff «L'Etoile» waren 8 Offiziere und 108 Matrosen auf einer Fläche von $30 \times 10$ Metern eingepfercht. Zu ihrem grossen Glück durfte sie mit Commerson die Kapitänskajüte bewohnen, die immerhin rund 40 Quadratmeter umfasste. Falls Gerüchte um ihre Identität aufgetaucht sein sollten, sind diese nirgends dokumentiert. Da sie kein Offizier war, wurde sie jedoch in die Mannschaftsunterkünfte verbannt. Sie konnte dort kein Auge zutun, da sie panische Angst vor all den Männern hatte. Eine Verletzung von Commerson durch einen früheren Hundebiss erlaubte es ihr, wieder in die Kapitänskajüte zurückzukehren, um ihn zu pflegen. Diese Verletzung (ein Ulcus cruris) machte ihm bis $\mathrm{zu}$ seinem Tod zu schaffen und verhinderte oft die Teilnahme an Landgängen. Um alle Gerüchte zu zerstreuen, verbreitete sie unter der Mannschaft die Botschaft, sie sei ein Eunuch. Das nächste Problem tauchte auf, als sie den Äquator überquerten. Befestigt an einem Seil, wurden alle Neulinge ins Meer getaucht - natürlich ohne Oberkleidung. Nur mit Mühe gelang es ihr, als Einzige bekleidet diese Äquator-Taufe zu erdulden. Ihre dauernden Bandagen verursachten am Oberkörper ein Ekzem. Die Beziehung zwischen Commerson und ihr war an einem Tiefpunkt angelangt, als sie nach langer Fahrt auf dem Atlantik Montevideo erreichten [10, S. 73-91].

\section{Botanische Exkursionen}

In der Umgebung von Rio gingen Baret und Commerson zum ersten Mal auf Pflanzenjagd. Da ihn sein Bein schmerzte, erledigte Jeanne Baret eigentlich die ganze Arbeit. Mehr als 60 Jahre später sollte dann ein gewisser Charles Darwin die gleiche Gegend erkunden! Baret und Commerson fingen auch viele Schmetterlinge und andere Insekten und pressten Pflanzen in einer tragbaren Pflanzenpresse. Die Schlepperei war für Baret eine grosse körperliche Belastung. Trotzdem genoss sie die Tage an Land. Währenddessen kamen Kapitän Bougainville die Verdächtigungen, Baret sei eine Frau, zu Ohren. Er liess sich aber vorderhand nichts anmerken und erlaubte weiterhin, dass sie Commerson als Assistent diente. Er und Commerson waren auf sie angewiesen, da sie ein grosses Wissen um mögliche nützliche Pflanzen hatte. Zudem musste Commerson eine Hilfe haben, da er allein nicht all die Geräte schleppen konnte. In Montevideo 
lernten sie Ilex paraguensis (Mate) kennen. Die Spanier hatten diese Pflanze allerdings schon früher entdeckt. Auch die vielen Sukkulenten, z.B. Opuntien, begeisterten die beiden Botaniker. Bald war die Kapitänskabine mit vielen Fundstücken ausgefüllt. Alles musste sicher verstaut und konserviert werden. Das Angebot, den Rest der Reise auf dem grösseren Schiff «La Boudeuse» zu verbringen, lehnte Commerson ab, da sein «Assistent» in diesem Fall in der Mannschaftskabine hätte leben müssen [10, S. 94-123].

\section{Sein Lastesel}

Die Durchfahrt durch die 330 Meilen der Magellanstrasse dauerte lange. Immerhin konnten die beiden Reisenden den einen oder anderen Landausflug zum Botanisieren machen. Bei ihren Ausflügen entdeckten sie unter anderem Heidelbeeren und den Sadebaum (Juniperus sabina). Baret war Commersons «Lastesel» und trug mehr, als ein Mann hätte tragen können. Der Schiffsarzt Vives hielt in seinem Tagebuch fest: «In Fainess ihr gegenüber muss ich festhalten, dass alle über ihre geleistete Arbeit staunten» [10, S. 133]. Baret fand einen grossen Reichtum an Farnen, Moosen und Flechten, aber auch an Blütenpflanzen wie z.B. Lilien, Anemonen oder Mohn. Oft begleitete sie der Prinz von NassauSiegen auf ihren Ausflügen. Er war der Einzige, der Baret mit Respekt begegnete und ihre Leistungen würdigte. Auch Seeelefanten und Pinguine entdeckten sie in Patagonien. Heilpflanzen fanden sie nur wenige, so z.B. Cyttaria darwinii, ein Pilz, der später nach Darwin benannt worden ist. 7 Wochen nach der Einfahrt in die Magellanstrasse erreichten sie den Pazifik [10, S. 127-148].

\section{Die Enttarnung}

Neun Wochen segelten sie in den Pazifik, ohne genaue Karten zu haben. In der Kapitänskabine hatten sie viel zu tun mit dem Trocknen und Ordnen der gefundenen Pflanzen. Die Kabine war ein «exotisches Kabinett», das ihr Schutz bot. Hier konnte Baret auch mal ihre Bandagen abnehmen. 1768 erreichten sie die ersten Inseln der Südsee. Nackte Männer in Pirogen näherten sich dem Schiff. Die Einheimischen merkten ihr sofort an, dass sie eine Frau war, und verrieten sie. Die anderen Schiffsleute mussten sie retten und in Sicherheit bringen. Schliesslich gab sie ihre Verkleidung zu. Der Schiffsarzt Vives hält in seinem Tagebuch die Enttarnung ebenfalls fest. Von Commerson ist dazu nichts geschrieben worden! Die Tahitianer waren fasziniert vom «Transvestit», der auch noch über Heilpflanzen Bescheid wusste. Einige Pflanzen wurden entdeckt, so z.B. die Brotfrucht (Artocarpus altilis) oder Ficus tinctoria (eine Färbepflanze). Mit dem offensichtlichen feigen Verrat von Commerson, der nicht zu ihr stehen wollte, verschlechterte sich ihre Beziehung massiv [10, S. 151-172].

\section{Die Hölle auf Erden}

Die Expedition erreichte Samoa. Die Melanesier zeigten sich ihnen gegenüber feindlich. Auf der Weiterfahrt hatte man zu wenig Proviant. Die ganze Mannschaft hungerte. Sowohl das Ekzem auf ihrer Brust als auch das Bein von Commerson machten ihnen grosse Probleme. In New Ireland wurde ihre Verkleidung, die bisher nur wenigen bekannt war, allen Matrosen offenkundig. Bei einem Landausflug wurde sie von Schiffsmatrosen vergewaltigt. Die Schuldigen wurden eruiert. Kapitän Bougainville zeigte sich wie Commerson als grosser Feigling, verschwieg die Vergewaltigung und bestrafte niemanden. Jeanne Baret zog sich in die Kabine zurück und zeigte sich für mehrere Wochen nicht mehr [10, S. 175-191].

\section{Das versprochene Land}

Mit Opium ruhiggestellt, blieb Baret wochenlang in ihrer Kabine. Sie litt, würden wir heute sagen, an einer posttraumatischen Belastungsstörung. Da Commerson sie dauernd pflegen musste, gab es keinen Botaniker, der auf angelaufenen Inseln etwas entdecken konnte. In fast 2 Jahren auf See hatte sie keinen Tag Privatsphäre gekannt. Nun musste sie sich Sorgen machen, was ihr die Zukunft noch bringen konnte. Bald merkte sie, dass sie schwanger war. Am 7. November 1768 erreichten sie Mauritius. Sie lernten den Botaniker Pierre Poivre kennen und entschieden sich, auf Mauritius zu bleiben, während die beiden Schiffe die Heimfahrt nach Frankreich antraten [10, S. 195-205].

Eigentlich war die Mission von Commerson ein Misserfolg, da er kaum kommerziell nutzbare Pflanzen auf der Reise entdeckt hatte. Er behielt den grössten Teil der gesammelten Pflanzen bei sich auf Mauritius. Die fortschreitende Schwangerschaft stellte für ihn ein Problem dar, war sie doch sehr wahrscheinlich eine Folge der Vergewaltigung. In Flacq gebar sie einen Sohn, den sie einer Familie zur Adoption überliess. Zusammen sammelten sie in den nächsten Jahren auf Mauritius mehr als 1000 Pflanzen. Ihre Beziehung beschränkte sich nun nur noch auf die Arbeit. Daneben führten sie ein getrenntes soziales Leben. 1770 nahmen sie an einer viermonatigen Expedition nach Madagaskar teil. Sie trug weiterhin männliche Kleider, auch wenn sie ihre Weiblichkeit nun nicht mehr versteckte. Auf Madagaskar benannte Commerson sogar eine neue Pflanzengattung nach ihr: Baretia. Der Name wurde später allerdings wieder geändert. Auch ein Abstecher auf La Réunion brachte viele neue Pflanzen. «Das Mädchen, das statistisch höchstens 20 Meilen von La Comelle entfernt sich aufhalten konnte, stand nun in Hosen auf einem aktiven Vulkan im Indischen Ozean» [10, S. 222]. Wieder auf Mauritius, lebten sie nun in Port Louis zum ersten Mal ruhig als Mann und Frau zusammen. Commerson starb 1773 an den Folgen 
der Beinverletzung, die er sich vor Jahren durch einen Hundebiss in Frankreich zugezogen hatte. Baret verlor damit einen Schüler, Lehrer, Mitarbeiter, Freund und Liebhaber. Die ganze Pflanzensammlung wurde per Schiff nach Paris zurückgeschickt. In den Augen des Gesetzes war sie eine einfache Hausangestellte. Sie besass nichts als ihre Kleider und ein grosses Wissen [10, S. 196228].

\section{Ein Denkmal, dauerhafter als eine Pyramide}

Immerhin vermachte Commerson ihr einen Teil seines Vermögens in Frankreich. Sie musste zuerst aber die Möglichkeit finden, nach Frankreich zurückzukehren. Sie heiratete 1774 einen Matrosen und kehrte mit ihm 1775 nach Frankreich zurück. Den Rest ihres Lebens verbrachte sie mit ihrem Ehemann in der französischen Dordogne. 1776 wurden ihr aus dem Vermächtnis von Commerson 600 Pfund übergeben. Vom Ministerium für Marine erhielt sie zudem eine jährliche Pension von 200 Pfund. Das erlaubte ihr den Kauf eines Hauses und ein sorgenfreies Leben. Ein Dokument aus dem MarineArchiv dokumentierte sogar ihre Leistungen. Somit erhielt sie doch eine gewisse Anerkennung. In den Aufzeichnungen der Weltumsegelung wird sie kaum erwähnt. Die Sammlung mit den Funden ihrer Reise vermoderte in Kisten, ohne dass sie je die Gelegenheit bekam, alles zu ordnen. Gewisse Stücke der Sammlung fanden Eingang in das Nationale Historische Museum und das Nationale Französische Herbar, allerdings ohne dass sie je erwähnt wird. Jeanne Baret bzw. Jeanne Dubernat starb am 5. August 1807 im Alter von 67 Jahren. Dank der Initiative der Biografin Glynis Ridley wurde vor Kurzem eine Pflanze zu ihren Ehren als Solanum baretiae (Abb. 2) bezeichnet [11].

\section{Unterdrückte Botanikerinnen}

Seit Menschengedenken waren Frauen als Heilkräuterkundige tätig - zuerst für ihre Familie und Sippe, später auch mit grösserem Wirkungskreis. Heute noch bekannt ist Hildegard von Bingen (1098-1179), die sich neben Musik und Religion auch mit Pflanzen befasste. Auch die Zubereitung der Nahrung, die bis weit in das 18. Jahrhundert als wesentlicher Teil der Medizin galt, war eine Domäne der Frauen. Mit dem Aufkommen von Medizin, Pharmazie und Botanik wurden diese Disziplinen zu Wissenschaften und gerieten ab Mitte des 18. Jahrhunderts unter männliche «Herrschaft». Im 18. Jahrhundert, mit dem Aufkommen der Wissenschaften und Universitäten, verwehrten die Männer den Frauen den Zugang. Einer der Hauptvertreter - Immanuel Kant - war sogar der Ansicht, die wissenschaftliche Ausbildung von Frauen laufe dem Naturgesetz zuwider. Jean-Jacques

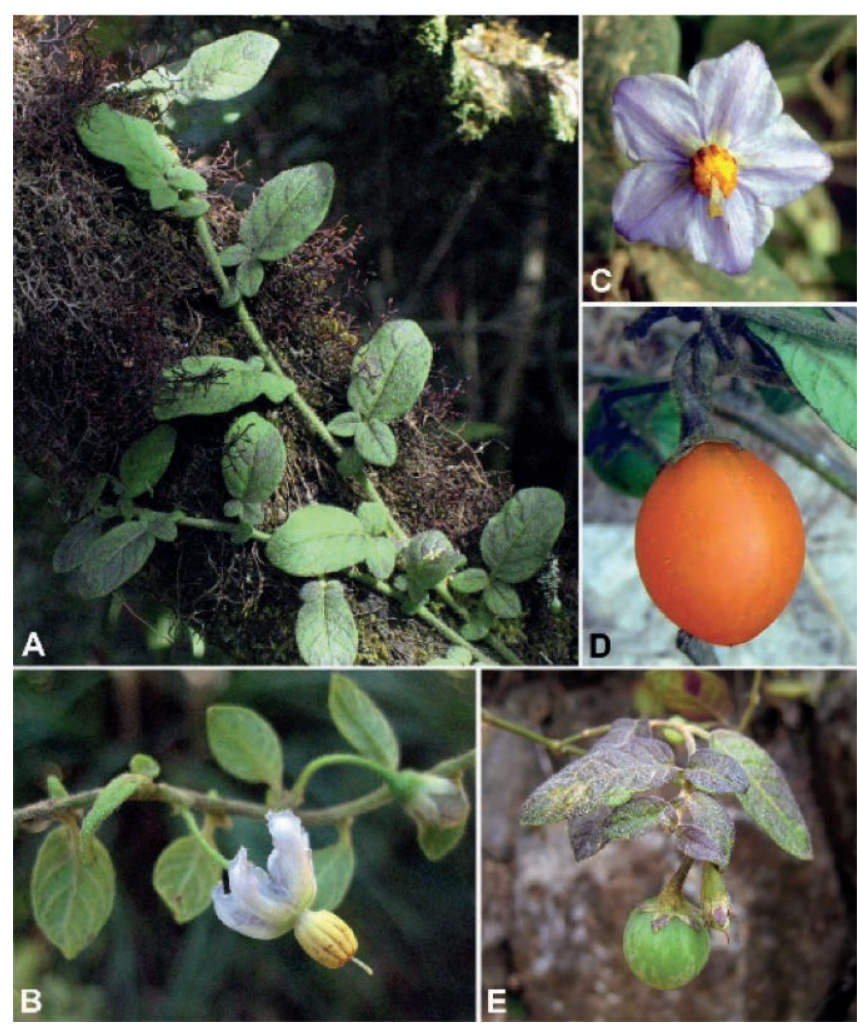

Abb. 2. Solanum baretiae [11].

Rousseau behauptete, die wissenschaftliche Tätigkeit erfordere ein Ausmass an Kraft, über das Frauen nicht verfügten. Den Einfluss der Frauen auf die Männer betrachtete er als unnatürlich, als Schaden für Körper und Geist [12, S. 372-389].

Mit Carl von Linné und seinem System der Botanik wurde die Macht der (männlichen) Wissenschaft weiter etabliert. In den aufkommenden Salons des 18. Jahrhunderts, wo literarische, künstlerische und wissenschaftliche Fragen diskutiert wurden, waren Frauen zum grössten Teil von der Teilnahme ausgeschlossen. Die moderne Botanik, früher als «Pflanzenkunde» noch vorwiegend Domäne der Frauen, geriet zunehmend unter männlichen Einfluss, wobei die Beschäftigung von Frauen mit Pflanzen sogar von Rousseau gefördert wurde, aber nicht im Sinne einer Wissenschaft, sondern als «Freizeitbeschäftigung». An den neu gegründeten Universitäten waren Frauen grösstenteils nicht zugelassen [12, S. 318-340].

Der wichtige Anteil der Frauen in der Naturwissenschaft wurde von den Männern zum Teil bewusst verleugnet. So konnte man sich auf einfache Art der Konkurrenz der Frauen entledigen. Trotzdem liessen sich viele Frauen nicht abschrecken und befassten sich mit Mathematik, Physik, Medizin oder auch Botanik. Bereits im 17. Jahrhundert erschienen erste wissenschaftliche Bücher und Zeitschriften von Frauen, z.B. von Aphra Behn (Astronomin), Elisabeth Carter (Physik) oder 
Louyse Bourgeois (Medizin). Zum Medizinstudium zugelassen wurden Frauen an den Universitäten aber erst Mitte des 19. Jahrhunderts [13, S. 110-136].

\section{Persönlichkeitsmerkmale der Botanikerinnen}

Vor diesem Hintergrund von Unterdrückung und Benachteiligung musste eine Frau, die sich im 18. Jahrhundert als Botanikerin betätigen wollte, bestimmte Persönlichkeitsmerkmale und Eigenschaften aufweisen. Dies wird im Folgenden anhand von sieben Botanikerinnen dargestellt [2, S. 34-37] (Abb. 3).

Drei Eigenschaften sind bei allen sieben Botanikerinnen prägnant vorhanden: Sie hatten zum Ersten eine starke Persönlichkeit mit viel Selbstvertrauen und einem grossen Willen. Zum Zweiten zeigten alle viel Enthusiasmus und Begeisterung für ihr Fachgebiet. Das machte sich oft schon in der Kindheit bemerkbar, indem sie sich intensiv mit Pflanzen und Natur beschäftigten. Ohne diese beiden Charakterzüge hätte sich kaum eine von ihnen in der von Männern dominierten Welt behaupten können. Die herausragende Figur ist hier sicher Jeanne Baret, die so viel Enthusiasmus und Mut besass, sich als Mann verkleidet auf eine Schiffsreise rund um die Welt zu begeben. Ohne ihre äusserst starke Persönlichkeit hätte sie dies kaum überlebt. Auch Maria Sibylla Merian ist hier besonders zu erwähnen, die mit ihrer Tochter, ohne einen männlichen «Beschützer», eine mehrjährige Expedition nach Surinam (Südamerika) unternahm. Zum Dritten war bei allen Botanikerinnen ein männlicher Förderer vorhanden. Während Jeanne Baret bei ihren Kräutersammlungen einem bekannten Botaniker begegnete, sind es bei den anderen Botanikerinnen der Vater, ein Verwandter oder der Ehemann, welche die botanischen Interessen der jungen Frauen förderten. Interessanterweise blieben drei Frauen ihr ganzes Leben lang unverheiratet. Von den anderen vier liessen sich zwei im Laufe ihres Lebens wieder scheiden - ebenfalls eher selten im 18. Jahrhundert.

Eine gute Bildung mit Privatunterricht zu Hause in verschiedenen Fächern war bei allen ausser bei Jeanne Baret vorhanden, wobei diese ein grosses Wissen $\mathrm{zu}$ Kräutern und Pflanzen besass, das sie wahrscheinlich von ihrer Mutter erwarb. Jeanne Baret war auch die Einzige, die nicht aus gutem Hause, d.h. nicht aus einer gutbürgerlichen oder sogar adligen Familie, stammte.

Richtig vermögend waren eigentlich nur zwei Frauen: Karoline Luise von Baden und Sophie Charlotte von Mecklenburg-Strelitz. Zwei andere Botanikerinnen glichen ihr fehlendes Vermögen durch eine grosse Geschäftstüchtigkeit aus: Maria Sibylla Merian betrieb verschiedene Tätigkeiten, um ihre teuren Publikationen und Reisen zu finanzieren. Elisabeth Blackwell verdiente sich ihr Geld mit Pflanzenzeichnungen [14]. Auch Anna Blackburne, Catharina Helena Dörrien und Karoline Luise von Baden waren begnadete Pflanzenzeichnerinnen. Von den beiden Letzten sind leider kaum Zeichnungen erhalten, weil diejenigen von Catharina Helena Dörrien nie publiziert wurden und die Originale in alle Welt verschwanden. Die Werke von Karoline Luise von Baden wurden aus Geldmangel ebenfalls nie publiziert und fielen im Zweiten Weltkrieg einem Brand zum Opfer.

Im Weiteren hatten alle Botanikerinnen mit Ausnahme von Jeanne Baret eine mehr oder weniger grosse Bekanntschaft und waren in Fachkreisen gut vernetzt.
Abb. 3. Merkmale der Botanikerinnen (dunkelgrün = prägnant; hellgrün = vorhanden; gelb = nicht bekannt; weiss $=$ nicht vorhanden).

\begin{tabular}{|c|c|c|c|c|c|c|c|}
\hline Kriterium & $\begin{array}{l}\text { Jeanne } \\
\text { Baret } \\
(1740- \\
1807)\end{array}$ & $\begin{array}{l}\text { Anna } \\
\text { Blackburne } \\
(1726- \\
1793)\end{array}$ & $\begin{array}{l}\text { Elizabeth } \\
\text { Blackwell } \\
(1700- \\
1758)\end{array}$ & $\begin{array}{l}\text { Catharina } \\
\text { Helena } \\
\text { Dörrien } \\
(1717- \\
1795)\end{array}$ & $\begin{array}{l}\text { Maria } \\
\text { Sibylla } \\
\text { Merian } \\
(1647- \\
1717) \\
\end{array}$ & $\begin{array}{l}\text { Karoline } \\
\text { Luise von } \\
\text { Baden } \\
(1723- \\
1783) \\
\end{array}$ & $\begin{array}{l}\text { Sophie } \\
\text { Charlotte von } \\
\text { Mecklenburg- } \\
\text { Strelitz (1744- } \\
1818 \text { ) }\end{array}$ \\
\hline \multicolumn{8}{|l|}{ Starke Persönlichkeit } \\
\hline \multicolumn{8}{|l|}{ Enthusiasmus } \\
\hline \multicolumn{8}{|l|}{ Männliche Förderer } \\
\hline \multicolumn{8}{|l|}{ Gute Bildung } \\
\hline \multicolumn{8}{|l|}{ Edle/gute Herkunft } \\
\hline \multicolumn{8}{|l|}{ «Gute» Heirat } \\
\hline \multicolumn{8}{|l|}{ Vermögen } \\
\hline \multicolumn{8}{|l|}{ Geschäftstüchtigkeit } \\
\hline \multicolumn{8}{|l|}{ Pflanzenzeichnerin } \\
\hline \multicolumn{8}{|l|}{ Breite Bekanntschaft } \\
\hline \multicolumn{8}{|l|}{ Forscherdrang } \\
\hline \multicolumn{8}{|l|}{ Publikationen } \\
\hline \multicolumn{8}{|l|}{ Reisen/Expeditionen } \\
\hline Anerkennung & & & & & & & \\
\hline
\end{tabular}


So standen z.B. Anna Blackburne und Karoline Luise von Baden mit dem berühmten Botaniker Carl von Linné in regem Kontakt. Der Forscherdrang und die wissenschaftliche Neugier sind bei vier der sieben Frauen klar dokumentiert. Allerdings absolvierte keine der sieben Botanikerinnen ein Studium oder eine Ausbildung in Botanik. Sie hatten sich ihr Wissen mehr oder weniger selber angeeignet und dann mit vielen Praxiserfahrungen erweitert und vertieft. Ihr Dilettantismus - im besten Sinne des Wortes «dilettare = sich erfreuen» - wurde einigen von ihnen später zum Vorwurf gemacht. Ihre Texte zu den Zeichnungen wurden als «unwissenschaftlich» kritisiert. Eine grosse Erschwernis für alle Frauen stellte die zum grössten Teil in Latein abgefasste Literatur zur Botanik dar. Nur von Karoline Luise von Baden und Anna Blackburne ist überliefert, dass sie des Lateins mächtig waren.

Publikationen waren damals sehr kostspielig, denn die Vorlagen mussten in Kupfer gestochen und von Hand koloriert werden. Die «erfolgreichste» unter den sieben Botanikerinnen war Maria Sibylla Merian. Sie stammte aus einer Druckerfamilie und lernte dieses Handwerk in ihrer Jugend. Mit geschickter Geschäftstätigkeit konnte sie verschiedene Bücher publizieren, die heute noch in Nachdrucken erhältlich sind [15]. Bei Sophie Charlotte von Mecklenburg-Strelitz, der Königin unter den Botanikerinnen, standen Publikationen nicht im Vordergrund. Sie legte dafür neben ihrer Bildersammlung ein umfangreiches Naturalienkabinett an. Trotz grosser Geschäftstüchtigkeit und Vermögen scheiterte Karoline Luise von Baden mit der grossartigen Idee, ein Werk mit Abbildungen aller von Linné entdeckten Pflanzen zu veröffentlichen. Catharina Helena Dörrien veröffentlichte vor allem pädagogische Werke und einen Katalog aller von ihr bestimmten Pflanzen von Oranien-Nassau. Sie war dabei eine der Ersten, die präzise Beschreibungen der Pflanzen in deutscher Sprache abfasste.
Grössere Reisen unternahmen nur vier der sieben Botanikerinnen. Bei Karoline Luise von Baden handelte es sich aber nicht um botanische Expeditionen, sondern um gesellschaftliche Reisen in europäische Städte, wo sie einen regen Austausch mit anderen Wissenschaftlern pflegte. Catharina Helena Dörrien blieb zeitlebens mit ihrer Heimat Oranien-Nassau verbunden und erkundete dabei alle dort wachsenden Pflanzen. Man kann sich vorstellen, wie oft sie in dem $60 \times 100 \mathrm{~km}$ grossen Gebiet unterwegs war, bis alle Pflanzen erfasst und beschrieben waren. Die Reise von Maria Sibylla Merian mit ihrer Tochter nach Surinam ist eine riesige Leistung, finanzierte sie diese Expedition doch selber und war mit über 50 Jahren für die damalige Zeit schon recht betagt. Zudem erkrankte sie an Malaria. Das Ergebnis dieser Reise war das Buch «Metamorphosis insectorum Surinamensium», das $1705 \mathrm{im}$ Format $50 \times 35 \mathrm{~cm}$ in einer lateinischen und einer holländischen Fassung im Eigenverlag erschien [16]. Das grösste Abenteuer erlebte aber Jeanne Baret, die von 1765-1775 als erste Frau um die Welt reiste. Sie hat leider keine eigenen Aufzeichnungen verfasst.

Alle Botanikerinnen bis auf Jeanne Baret fanden bereits in ihrer Zeit wenigstens eine gewisse Anerkennung: Anna Blackburne galt als anerkanntes Mitglied der wissenschaftlichen Gemeinschaft, Catharina Helena Dörrien wurde Mitglied verschiedener botanischer Gesellschaften, Maria Sibylla Merian wurde berühmt mit ihren speziellen Zeichnungen von Insekten und Pflanzen, und Sophie Charlotte von Mecklenburg-Strelitz erregte Aufsehen mit ihrem grossen Naturalienkabinett. Von Jeanne Baret sind mit Ausnahme der Pflanzenliste, die ihrem Begleiter Commerson zugeschrieben wurde, keinerlei Aufzeichnungen vorhanden.

\section{Disclosure Statement}

Der Autor gibt an, dass im Zusammenhang mit dem Manuskript keine Interessenkonflikte bestehen.

\section{Literatur}

1 Weimer S: Die Botanikerin, welche die Welt als Mann verkleidet umsegelte. Zürich, Tagesanzeiger, 07.01.2012.

2 www.weiterbildung.uzh.ch/programme/ ethnobot/Abschlussarbeiten/ PionierinnenBotanikerinnen.pdf.

3 Schubert R, Wagner G: Botanisches Wörterbuch. Stuttgart, Ulmer, 2000.

4 http://de.wikipedia.org/wiki/Geschichte_ der_Botanik_(Zeitstrahl).

5 www.padovaincoming.it/info/ padua-stadt-wissenschaft/de.

6 http://de.wikipedia.org/wiki/Frauenstudium.

7 http://de.wikipedia.org/wiki/Kategorie: Botaniker_(18._Jahrhundert).
8 Viereck R: «Zwar sind es weibliche Hände» - Die Botanikerin und Pädagogin Catharina Helen Dörrien 1717-1795. Frankfurt/M., Campus, 2000.

9 Lauts J: Karoline Luise von Baden. Karlsruhe, C.F. Müller, 1980.

10 Ridley G: The Discovery of Jeanne Baret a Story of Science, the High Seas, and the First Woman to Circumnavigate the Globe. New York, Broadway Paperbacks, 2010.

-11 Tepe EJ, Ridley G, Bohs L: A new species of Solanum named for Jeanne Baret, an overlooked contributor to the history of botany. PhytoKeys 2012;8:37-47. www.pensoft.net/ journals/phytokeys/article/2101/a-newspecies-of-solanum-named-for-jeanne- baret-an-overlooked-contributor-to-thehistory-of-botany.

12 Schiebinger L: Schöne Geister - Frauen in den Anfängen der modernen Wissenschaft. Stuttgart, Klett-Cotta, 1993.

13 Alic M: Hypatias Töchter - Der verleugnete Anteil der Frauen an der Naturwissenschaft. Zürich, Union, 1987.

14 Heilmann P: Das Kräuterbuch der Elisabeth Blackwell. Dortmund, Harenberg, 1984.

15 Wettengl K (Hrsg): Maria Sibylla Merian: 1647-1717; Künstlerin und Naturforscherin. Ostfildern, Hatje Cantz, 1997.

16 Schubert K: Maria Sibylla Merian - Reise nach Surinam. München, Frederking und Thaler, 2010. 